\title{
INEQUALITIES SATISFIED BY ENTIRE FUNCTIONS AND THEIR DERIVATIVES
}

BY

\author{
BOO SANG LEE AND S. M. SHAH( $\left.{ }^{(}\right)$
}

\begin{abstract}
For a class of entire functions with simple and positive zeros, it is shown that the maximum of the moduli of the first two Taylor coefficients at any point $z$, dominate all the remaining Taylor coefficients, provided $|z|$ is sufficiently large. Further, there is a subclass for which this result holds at every point $z$.
\end{abstract}

1. Introduction. In this paper we shall be concerned with inequalities satisfied by the derivatives of entire functions with simple zeros. Let $\left\{a_{n}\right\}_{1}^{\infty}$ be a sequence of positive numbers such that

$$
a_{n+1} / a_{n} \equiv \alpha_{n} \geqq \gamma>1
$$

and let

$$
P(z)=\prod_{1}^{\infty}\left(1-\frac{z}{a_{n}}\right)
$$

We prove

THEOREM 1. Suppose $P(z)$ is an entire function given by (1.2) where the zeros $a_{n}$ satisfy (1.1). Then for a given number $M>0$, there exists a number $R=R(M)$ such that for every $z,|z|>R$,

$$
\max \left\{|P(z)|,\left|P^{\prime}(z)\right|\right\}>M\left|P^{(j)}(z)\right| / j !, \quad j=2,3, \ldots
$$

THEOREM 2. Let $P(z)$ be given by (1.2). If

$$
\alpha_{n} \geqq 3, \quad n=2,3, \ldots, \quad a_{2}-a_{1} \geqq 8,
$$

then for every $z$

$$
\max \left\{|P(z)|,\left|P^{\prime}(z)\right|\right\}>\left|P^{(j)}(z)\right| / j !, \quad j=2,3, \ldots
$$

THEOREM 3. Let $\left\{\psi_{n}\right\}_{1}^{\infty}$ be a sequence of positive numbers. Then there exists an entire function $Q(z)$ such that for every $z$

$$
\max \left\{|Q(z)|,\left|Q^{\prime}(z)\right|\right\}>\psi_{j-1}\left|Q^{(j)}(z)\right|, \quad j=2,3, \ldots
$$

Presented to the Society, January 23, 1970; received by the editors August 15, 1969.

AMS Subject Classifications. Primary 3055; Secondary 3057, 3058.

Key Words and Phrases. Entire functions, canonical products, functions of bounded index.

( $\left.{ }^{1}\right)$ The research of this author was supported by the National Science Foundation under grant GP-7544.

Copyright (C) 1970, American Mathematical Society 
THEOREM 4. Let $P(z)$ be given by (1.2) where $a_{n+1} / a_{n} \geqq n+1,(n \geqq 1), a_{2}-a_{1} \geqq 11$ and $a_{1}>0$. Then for every $z$

$$
\max \left\{|P(z)|,\left|P^{\prime}(z)\right|\right\}>\phi_{j-1}\left|P^{(j)}(z)\right|, \quad j=2,3, \ldots
$$

where $\phi_{n} \geqq 1, n \geqq 1$ and $\phi_{n} \rightarrow \infty$ as $n \rightarrow \infty$.

2. Preliminary results. In this section we suppose that (1.1) holds and obtain bounds for zeros $\left\{b_{n}\right\}_{1}^{\infty}$ of $P^{\prime}(z)$. By Laguerre's theorem [1, p. 23], [7, p. 266] we know that the $b_{n}$ 's are all real and $a_{1}<b_{1}<a_{2}<\cdots<a_{n}<b_{n}<a_{n+1}<\cdots$. Let

$$
h(t)=\sum_{1}^{\infty} \frac{1}{t^{j}-1}, \quad t>1 .
$$

Then $h(t)$ is decreasing in $(1, \infty)$ and $h(2.8)<0.778$.

LEMMA 1. Let $q$ be the smallest integer such that $q \geqq h(\gamma)+\gamma /(\gamma-1)$. Then

$$
\begin{aligned}
\quad b_{n}<\left(n a_{n+1}+a_{n}\right) /(n+1), & n=1,2, \ldots, \\
\{1-1 /(n-h(\gamma))\} a_{n+1}<b_{n}, & n=q+1, q+2, \ldots,
\end{aligned}
$$

and

$$
b_{n+1} / b_{n} \equiv \beta_{n} \geqq \gamma^{\prime}>1, \quad n=1,2, \ldots
$$

Proof. (i) Let $x=\left(n a_{n+1}+a_{n}\right) /(n+1)$. Then

$$
\frac{P^{\prime}(x)}{P(x)}=\sum_{1}^{\infty} \frac{1}{x-a_{j}}<\frac{n}{x-a_{n}}+\frac{1}{x-a_{n+1}}=0,
$$

and (2.1) is proved.

(ii) Let $x=\{1-1 /(n-h(\gamma))\} a_{n+1}, n \geqq q+1$. By our choice of $q, a_{n}<x<a_{n+1}$ and

$$
\sum_{n+2}^{\infty} \frac{1}{x-a_{j}}>-\frac{1}{a_{n+1}} \sum_{j=1}^{\infty} \frac{1}{\gamma^{j}-1}=\frac{-h(\gamma)}{a_{n+1}} \text {. }
$$

Hence

$$
\frac{P^{\prime}(x)}{P(x)}>\frac{n-1-h(\gamma)}{a_{n+1}}+\frac{1}{x-a_{n}}+\frac{1}{x-a_{n+1}}>0,
$$

and (2.2) is proved.

(iii) From (2.1) and (2.2) we have, for $n \geqq q+1$,

$$
\frac{b_{n+1}}{b_{n}} \geqq \frac{n-h(\gamma)}{n+1-h(\gamma)} \frac{(n+1) a_{n+2}}{n a_{n+1}+a_{n}}>\gamma \frac{n-h(\gamma)}{n+1-h(\gamma)} .
$$

Let $\gamma=1+c$. Then $c>0$. Let $c^{\prime}=c /(2 \gamma)<1$. Let $t$ be an integer such that

$$
t>\max \left\{q+1,1 / c^{\prime}-1+h(\gamma)\right\} \text {. }
$$

Then, for all $n \geqq t$,

$$
\frac{b_{n+1}}{b_{n}}>\gamma \frac{n-h(\gamma)}{n+1-h(\gamma)}>\left(1-c^{\prime}\right) \gamma>1 \text {. }
$$

Let $\gamma^{\prime}=\min \left\{\beta_{1}, \beta_{2}, \ldots, \beta_{t-1},\left(1-c^{\prime}\right) \gamma\right\}$. Then $\beta_{n} \geqq \gamma^{\prime}>1$ for $n \geqq 1$. 
LEMMA 2. Let $\rho>0$ and let

$$
U_{n}(\rho)=\bigcup_{j=n}^{\infty}\left\{z|| z-a_{j} \mid \leqq \rho\right\}, \quad V_{n}(\rho)=\bigcup_{j=n}^{\infty}\left\{z|| z-b_{j} \mid \leqq \rho\right\} .
$$

Then there exists an integer $M_{0}$ such that $U_{M_{0}}(\rho) \cap V_{M_{0}}(\rho)=\varnothing$.

Proof. Let $M_{1} \geqq 2$ be an integer such that

$$
M_{1}+1 \geqq \frac{1}{\log \gamma}, \quad \frac{\gamma^{M_{1}+1}}{M_{1}+1}>\frac{2 \rho \gamma^{3}}{(\gamma-1) a_{2}} .
$$

Since $\gamma^{x} / x$ is an increasing function of $x$ on $[1 / \log \gamma, \infty)$ we have, for $n \geqq M_{1}$,

$$
\frac{a_{n+1}-a_{n}}{n+1} \geqq \frac{\gamma^{n-2}(\gamma-1) a_{2}}{n+1}>2 \rho .
$$

Let $M_{2} \geqq q+2$ be such that for $n \geqq M_{2}$

$$
\gamma \frac{1-1 /(n-h(\gamma))-1 /(n+1)}{1-1 /(n+1)}>1 \text {. }
$$

Then for all $n \geqq M_{0}=\max \left(M_{1}, M_{2}\right)$

$$
\begin{gathered}
b_{n}-a_{n}>\left\{1-\frac{1}{n-h(\gamma)}\right\} a_{n+1}-a_{n}>\frac{a_{n+1}-a_{n}}{n+1}>2 \rho, \\
a_{n+1}-b_{n}>\frac{a_{n+1}-a_{n}}{n+1}>2 \rho
\end{gathered}
$$

and the lemma is proved.

LEMMA 3. Let $M \geqq 1$. Then for any number $\rho>2 M$ there exists an integer $N_{0}>0$ such that for every $z \notin U_{N_{0}}(\rho)$ and $|z|>a_{N_{0}}$

$$
|P(z)|>M\left|P^{(j)}(z)\right| / j !, \quad j=1,2, \ldots
$$

Proof. Let

$$
G(z)=\sum_{1}^{\infty} \frac{1}{z-a_{j}}, \quad \sigma=\sum_{1}^{\infty} \frac{1}{\left|z-a_{j}\right|}, \quad z \notin U_{1}(\rho) .
$$

Then

$$
P^{\prime}(z) / P(z)=G(z)
$$

and

$$
\frac{P^{(k+1)}(z)}{(k+1) !}=\frac{1}{k+1} \sum_{j=0}^{k} \frac{G^{(j)}(z)}{j !} \frac{P^{(k-j)}(z)}{(k-j) !} .
$$

Hence

$$
\begin{aligned}
\frac{\left|P^{(k+1)}(z)\right|}{(k+1) !} & \leqq \frac{1}{k+1}\left(\sum_{i=0}^{k} \frac{\left|G^{(i)}(z)\right|}{i !}\right) \max _{0 \leqq i \leqq k}\left\{\frac{\left|P^{(i)}(z)\right|}{i !}\right\} \\
& \leqq \sigma \max _{0 \leqq i \leqq k}\left\{\frac{\left|P^{(i)}(z)\right|}{i !}\right\}, \quad k=0,1, \ldots
\end{aligned}
$$


We estimate $\sigma$. Write $z=x+i y$ and let $a_{n}<x \leqq a_{n+1}, n \geqq 1$. Since $z \notin U_{1}(\rho)$ we have

$$
\sigma \leqq \frac{n-1}{a_{n}-a_{n-1}}+\frac{2}{\rho}+\frac{h(\gamma)}{a_{n+1}}
$$

Here $a_{0}=0$. Write $c_{1}=(\rho-2 M) / 2, c_{2}=c_{1} / M\left(2 M+c_{1}\right)$. Choose $N=N\left(c_{1}\right)$ such that for $n \geqq N$

$$
n /\left(a_{n}-a_{n-1}\right)+h(\gamma) / a_{n}<c_{2}
$$

Then

$$
\sigma<c_{2}+2 / \rho<1 / M,
$$

and so for every $z$ with $z \notin U_{N}(\rho)$ and $x>a_{N}$, we have from (2.8)

$$
\max _{0 \leqq i \leqq k}\left\{\frac{\left|P^{(i)}(z)\right|}{i !}\right\}>M \frac{\left|P^{(k+1)}(z)\right|}{(k+1) !}, \quad k=0,1, \ldots
$$

Let $S=\{z \mid x \leqq 0\} \cup\left\{z\left|0<x \leqq a_{N},\right| y \mid \geqq a_{N}\right\}$. Then, for $z \in S$,

$$
\sigma<\frac{N}{a_{N}}+\sum_{j=1}^{\infty} \frac{1}{\left(\gamma^{j}-1\right) a_{N}}<\frac{h(\gamma)+N}{a_{N}}<c_{2}<\frac{1}{M} .
$$

Finally choose integer $N_{0}$ such that $a_{N_{0}} \geqq \sqrt{ } 2 a_{N}$. Then for every $z \notin U_{N_{0}}(\rho)$ and $|z|>a_{N_{0}}$, (2.10) holds and this implies (2.4).

3. Proof of Theorem 1. We have $P^{\prime}(z)=P^{\prime}(0) \prod_{1}^{\infty}\left(1-z / b_{n}\right)$ where, by (2.3), $\beta_{n} \equiv b_{n+1} / b_{n} \geqq \gamma^{\prime}>1$. Hence we can apply the argument used to prove (2.4) to $P^{\prime}(z)$, and obtain that there exists $N_{1}$ such that for $z \notin V_{N_{1}}(\rho)$ and $|z|>b_{N_{1}}$

$$
\left|P^{\prime}(z)\right|>M \frac{\left|P^{(k+1)}(z)\right|}{k !}>M \frac{\left|P^{(k+1)}(z)\right|}{(k+1) !}, \quad k=1,2, \ldots
$$

By Lemma 2, we can choose $N_{2} \geqq \max \left(N_{0}, N_{1}\right)$ such that $U_{N_{2}}(\rho) \cap V_{N_{2}}(\rho)=\varnothing$. Write $R=b_{N_{2}}$. Then for every $z,|z|>R,(1.3)$ holds and the theorem is proved.

4. Proof of Theorem 2. We first prove two lemmas wherein, and also in the proof of Theorem 2, we follow the notation of $\$ 2$.

LEMMA 4. (i) If $\alpha_{n} \geqq 2.8$ for $n \geqq 2$, then

$$
b_{n}>x_{n} \equiv \frac{n a_{n+1}+2 a_{n}}{n+2} \quad(n \geqq 1) .
$$

(ii) If $\alpha_{n} \geqq 3$ for $n \geqq 2$, then

$$
\beta_{n} \geqq 2.8 \quad(n \geqq 2) .
$$

(iii) If $\alpha_{n} \geqq n+1$ for $n \geqq 1$, then

$$
\beta_{n} \geqq n+1 \quad(n \geqq 1) .
$$


Proof of Lemma 4. Since $a_{n}<x_{n}<a_{n+1}$, we have

$$
\begin{aligned}
\frac{P^{\prime}(x)}{P(x)} & >\sum_{j=1}^{n} \frac{1}{x_{n}-a_{j}}-\frac{h(2.8)}{a_{n+1}} \\
& \geqq \frac{n-1-h(\gamma)}{a_{n+1}}-\frac{n^{2}-4}{2 n\left(a_{n+1}-a_{n}\right)} .
\end{aligned}
$$

For $n \geqq 6$, the last expression is nonnegative and for $1 \leqq n \leqq 5$, the first expression is easily seen to be nonnegative. Hence (4.1) is proved.

Next we have, by (4.1) and (2.1), for $n \geqq 1$,

$$
\frac{b_{n+1}}{b_{n}} \geqq \frac{2 a_{n+1}+(n+1) a_{n+2}}{a_{n}+n a_{n+1}} \frac{(n+1)}{(n+3)} \text {. }
$$

Simple computation now yields (4.2) and (4.3).

LemmA. 5. Let $\rho=(3-\sqrt{ } 5)\left(a_{2}-a_{1}\right) / 4$ and $A=7.5 /\left(a_{2}-a_{1}\right)$. Suppose $\alpha_{n} \geqq 2.8$ for $n \geqq 2$. Then

$$
U_{1}(\rho) \cap V_{1}(\rho)=\varnothing,
$$

and for all $z \notin U_{1}(\rho)$

$$
\sigma<A \text {. }
$$

Proof. By a simple computation, we have

$$
a_{1}+2 \rho<b_{1}<a_{2}-2 \rho,
$$

and $a_{n}+6\left(a_{2}-a_{1}\right) / 10<b_{n}<a_{n+1}-6\left(a_{2}-a_{1}\right) / 10,(n \geqq 2)$. Now (4.4) follows from the fact that $\rho<2\left(a_{2}-a_{1}\right) / 10$.

To prove (4.5) we first note that for $z \notin U_{1}(\rho)$ and $a_{n}<x \leqq a_{n+1},(n \geqq 2)$,

$$
\sigma \leqq \frac{n-1}{a_{n}-a_{n-1}}+\frac{1}{\rho}+\frac{1}{a_{n+1}-a_{n}-\rho}+\frac{h(2.8)}{a_{n+1}} .
$$

For $z \notin U_{1}(\rho)$ and $a_{1}<x \leqq a_{2}$ we have

$$
\sigma \leqq 1 / \rho+1 /\left(a_{2}-a_{1}-\rho\right)+h(2.8) / a_{2} .
$$

Finally for $z \notin U_{1}(\rho)$ and $x \leqq a_{1}$

$$
\sigma \leqq 1 / \rho+1 /\left(a_{2}-a_{1}\right)+h(2.8) / a_{2} .
$$

Hence for $z \notin U_{1}(\rho)$, either (4.7) or (4.8) holds. In either case $\sigma<A$ and (4.5) is proved.

Proof of Theorem 2. Since $\alpha_{n} \geqq 3$ and $a_{2}-a_{1} \geqq 8$ we have, by Lemma $5, \sigma<A<1$. Hence (2.5) and (2.8) show that for $z \notin U_{1}(\rho)$,

$$
\left|P^{(k+1)}(z)\right| /(k+1) !<\max _{0 \leqq i \leqq k}\left\{\left|P^{(i)}(z)\right| / i !\right\}, \quad k=0,1, \ldots
$$

This gives for $z \notin U_{1}(\rho),\left|P^{(k)}(z)\right||k !<| P(z) \mid, k=1,2, \ldots$ 
Next we consider $P^{\prime}(z)=P^{\prime}(0) \prod_{1}^{\infty}\left(1-z / b_{n}\right)$ and note that $\beta_{n} \geqq 2.8$, for $n \geqq 2$, by Lemma 4. Hence Lemma 5 applied to $P^{\prime}(z) \mid P^{\prime}(0)$ for $z \notin V_{1}(\rho)$ gives that

$$
\sigma^{\prime} \equiv \sum_{1}^{\infty} \frac{1}{\left|z-b_{n}\right|}<A
$$

Consequently, for $z \notin V_{1}(\rho)$

$$
\left|P^{\prime}(z)\right|>\frac{\left|P^{(k+1)}(z)\right|}{k !}>\frac{\left|P^{(k+1)}(z)\right|}{(k+1) !}, \quad k=1,2, \ldots
$$

Since $U_{1}(\rho) \cap V_{1}(\rho)=\varnothing$, the inequality (1.5) holds for every $z$.

5. Proof of Theorem 3. We need two lemmas.

Lemma 6. Suppose $P(z)$ is defined by (1.2) where $a_{n+1} / a_{n} \geqq n+1, n=2,3, \ldots$, $a_{2}>a_{1}>0$. Write $B=\max \left(A, 2 A^{2}\right)$ where $A=(7.5) /\left(a_{2}-a_{1}\right)$. Then, for every $z$, $B \max \left\{|P(z)|,\left|P^{\prime}(z)\right|\right\}>\left|P^{\prime \prime}(z)\right|$.

Proof. For $z \notin U_{1}(\rho)$, we have, by (2.6), $\left|P^{\prime}(z)\right|=|P(z) G(z)| \leqq \sigma|P(z)|$ and $\left|P^{\prime \prime}(z)\right|$ $=\left|P(z)\left\{G^{2}(z)+G^{\prime}(z)\right\}\right|<2 \sigma^{2}|P(z)|$. Now $\alpha_{n} \geqq 3$ for $n \geqq 2$. Hence by Lemma $5, \sigma<A$. It follows that for every $z \notin U_{1}(\rho)$

$$
\left|P^{\prime}(z)\right|<B|P(z)|, \quad\left|P^{\prime \prime}(z)\right|<B|P(z)| .
$$

By Lemma $4, \beta_{n} \geqq n+1 \geqq 3$ for $n \geqq 2$. Hence the above argument may be applied to $P^{\prime}(z) / P^{\prime}(0)$. By (4.9) we have for every $z \notin V_{1}(\rho)$

$$
\left|P^{\prime \prime}(z)\right|<B\left|P^{\prime}(z)\right|, \quad\left|P^{\prime \prime}(z)\right|<B\left|P^{\prime}(z)\right| .
$$

Since $U_{1}(\rho) \cap V_{1}(\rho)=\varnothing$, the lemma follows from (5.1) and (5.2).

LeMma 7. Let $P(z)$ be defined by (1.2) and suppose that

$$
a_{n+1} / a_{n} \geqq n+1, \quad(n=2,3, \ldots), a_{2}>a_{1}>0 .
$$

Denote the zeros of $P^{(k)}(z)$ by $\left\{a_{n}^{(k)}\right\}_{1}^{\infty}$ with $a_{n+1}^{(k)}>a_{n}^{(k)}$. Then

$$
a_{2}^{(k)}-a_{1}^{(k)} \geqq(3 / 2)^{k}\left(a_{2}^{(0)}-a_{1}^{(0)}\right),
$$

where $a_{n}^{(0)} \equiv a_{n}$.

Proof. By (2.1) and (4.1) we have

$$
a_{2}^{(1)}-a_{1}^{(1)}>\left(a_{3}-a_{1}\right) / 2>3\left(a_{2}-a_{1}\right) / 2 .
$$

An induction argument completes the proof of the lemma.

Proof of Theorem 3. We construct a new sequence $\left\{a_{n}\right\}_{1}^{\infty}$ as follows:

$$
\begin{aligned}
a_{1} & \geqq \max \left\{15,8 \psi_{1}\right\}, \quad a_{2} \\
a_{n+1} & \geqq \max \left\{(n+1) a_{n}, 4(n+1)(n+2)\left(\psi_{n+1}, 24 \psi_{n-1}\right)\right\}, \quad n=2,3, \ldots .
\end{aligned}
$$

Let $Q(z)=\prod_{1}^{\infty}\left(1-z / a_{n}\right)$. Since $a_{2}-a_{1} \geqq 15$ we have in Lemma $6 B=A<8 / a_{1}$. Hence for every $z$ 


$$
\left(8 / a_{1}\right) \max \left\{|Q(z)|,\left|Q^{\prime}(z)\right|\right\}>\left|Q^{\prime \prime}(z)\right| \text {. }
$$

Denote the zeros of $Q^{(k)}(z)$ by $\left\{a_{n}^{(k)}\right\}_{1}^{\infty}$. Then $a_{n}^{(0)}=a_{n}$, and by (4.3) and (5.3) we have

$$
a_{n+1}^{(k)} / a_{n}^{(k)} \geqq n+1, \quad(n \geqq 1), \quad a_{2}^{(k)}-a_{1}^{(k)} \geqq 15 .
$$

Hence (5.5) holds for $Q^{(k)}(z)$. Thus we have for every $z$ and $k \geqq 0$

$$
\left(8 / a_{1}^{(k)}\right) \max \left\{\left|Q^{(k)}(z)\right|,\left|Q^{(k+1)}(z)\right|\right\}>\left|Q^{(k+2)}(z)\right| .
$$

By (4.1) we have

$$
a_{n}^{(j+1)}>(n /(n+2)) a_{n+1}^{(j)}, \quad j \geqq 0, \quad n \geqq 1,
$$

and hence

$$
a_{n}^{(j)}>\frac{n(n+1)}{(n+j)(n+j+1)} a_{n+j}
$$

In particular,

$$
a_{1}^{(j)}>\frac{2}{(j+1)(j+2)} a_{j+1}, \quad j \geqq 1 .
$$

From (5.6) and (5.7)' we have for every $z$ and $k \geqq 0$

$$
\left|Q^{(2 k+2)}(z)\right|<\left\{(2 k+2) ! 4^{(k+1)} /\left(\prod_{j=0}^{k} a_{2 j+1}\right)\right\} \max \left\{|Q(z)|,\left|Q^{\prime}(z)\right|\right\} .
$$

Similarly we obtain for every $z$ and $k \geqq 0$

$$
\left|Q^{(2 k+3)}(z)\right|<\left\{(2 k+3) ! 4^{k+1} /\left(\prod_{j=0}^{k} a_{2 j+2}\right)\right\} \max \left\{|Q(z)|,\left|Q^{\prime}(z)\right|\right\} .
$$

Since $\prod_{j=0}^{k} a_{2 j+1} \geqq 4^{k+1}(2 k+2) ! \psi_{2 k+1}$, we have from (5.8) that for every $z$ and $k \geqq 0$

$$
\psi_{2 k+1}\left|Q^{(2 k+2)}(z)\right|<\max \left\{|Q(z)|,\left|Q^{\prime}(z)\right|\right\} .
$$

Similarly from (5.9) we have that for every $z$ and $k \geqq 0$

$$
\psi_{2 k+2}\left|Q^{(2 k+3)}(z)\right|<\max \left\{|Q(z)|,\left|Q^{\prime}(z)\right|\right\} .
$$

The theorem now follows from (5.10) and (5.11).

6. Proof of Theorem 4. We first prove

LEMMA 8. Let $P(z)$ be defined by (1.2) and suppose that $a_{n+1} / a_{n} \geqq n+1,(n \geqq 1)$, $a_{1} \geqq 15$. Then there exists a sequence $\left\{\lambda_{n}\right\}_{1}^{\infty}$ such that

$$
\lambda_{n}>1, \quad(n \geqq 1), \quad \lambda_{n} \rightarrow \infty \quad \text { as } n \rightarrow \infty ;
$$

and such that for every $z$

$$
\max \left\{|P(z)|,\left|P^{\prime}(z)\right|\right\}>\lambda_{j-1}\left|P^{(j)}(z)\right|, \quad j=2,3, \ldots .
$$


Proof. Define a sequence $\left\{\lambda_{n}\right\}_{1}^{\infty}$ as follows:

$$
\lambda_{1}=15 / 8, \quad \lambda_{2}=\frac{2 a_{1}}{24}, \quad \lambda_{n+1}=\frac{a_{n} \lambda_{n-1}}{4(n+2)} \quad(n \geqq 2) .
$$

If we replace $\left\{\psi_{n}\right\}_{1}^{\infty}$ by $\left\{\lambda_{n}\right\}_{1}^{\infty}$ in (5.4), the argument used in the proof of Theorem 3 gives (6.2). To prove (6.1) we note that $\lambda_{1}>1, \lambda_{2}>1$. For $k \geqq 1$ we have

$$
\lambda_{2 k+2}>((2 k) ! /(k+1) !) 2^{k} \text {, and } \lambda_{2 k+1}>((2 k-1) ! /(k+1) !) 2^{k} \text {. }
$$

These inequalities prove (6.1) and the lemma.

Proof of Theorem 4. We consider $P^{(3)}(z)$ and note that $a_{n+1}^{(3)} / a_{n}^{(3)} \geqq n+1(n \geqq 1)$, $a_{1}^{(3)} \geqq 15$. By Lemma 8 we can find a sequence $\left\{\lambda_{n}\right\}_{1}^{\infty}$ such that $\lambda_{n}>1,(n \geqq 1), \lambda_{n} \rightarrow \infty$ as $n \rightarrow \infty$ and such that for every $z$

$$
\max \left\{\left|P^{(3)}(z)\right|,\left|P^{(4)}(z)\right|\right\}>\lambda_{j-1}\left|P^{(j+3)}(z)\right|, \quad j=2,3, \ldots
$$

Since $a_{2}-a_{1} \geqq 11$, the constant $B$ in Lemma 6 is less than unity and hence we have, for every $z, \max \left\{|P(z)|,\left|P^{\prime}(z)\right|\right\}>\left|P^{\prime \prime}(z)\right|$. By Lemma 7 we have, for every $z$, $\max \left\{\left|P^{\prime}(z)\right|,\left|P^{\prime \prime}(z)\right|\right\}>\left|P^{m}(z)\right|$ and so on. Consequently, we have for every $z$, $\max \left\{|P(z)|,\left|P^{\prime}(z)\right|\right\}>\left|P^{(j)}(z)\right|, j \geqq 2$; and, in particular,

$$
\max \left\{|P(z)|,\left|P^{\prime}(z)\right|\right\}>\max \left\{\left|P^{(3)}(z)\right|,\left|P^{(4)}(z)\right|\right\} .
$$

Now define

$$
\begin{array}{ll}
\phi_{j}=1, & j=1,2,3, \\
\phi_{j}=\lambda_{j-3}, & j \geqq 4 .
\end{array}
$$

Then (6.3), (6.4) and (6.5) yield Theorem 4.

7. Remarks. (i) A transcendental entire function $f(z)$ is said to be of bounded index if there exists an integer $N \geqq 0$ such that for all $z$

$$
\max _{0 \leqq k \leqq N}\left\{\left|f^{(k)}(z)\right| / k !\right\} \geqq\left|f^{(j)}(z)\right| / j !, \quad j=1,2, \ldots ;
$$

and the smallest such integer $N$ is called the index of $f(z)$ (cf. [2], [3], [5]).

We have proved in Theorem 1 that (7.1), with $f$ replaced by $P$, holds for all $z$ such that $|z|>R$ with $N=1$. Now it is known that (cf. [6, pp. 132-133]) there exists an integer $N$ such that (7.1), with $f$ replaced by $P$, holds for all $z$ with $|z| \leqq R$. Thus we conclude that $P(z)$ is of bounded index.

(ii) Pugh and Shah [4] have shown that if $\left\{z_{n}\right\}_{1}^{\infty}$ is any sequence of complex numbers such that

$$
\left|z_{n+1}\right| \geqq 5^{n}\left|z_{n}\right|, \quad\left|z_{1}\right| \geqq 5,
$$

then the derivatives $f^{(j)}(z)$ of the corresponding canonical product $f(z)$ satisfy for all $z$,

$$
\max \left\{|f(z)|,\left|f^{\prime}(z)\right|\right\}>\left|f^{(j)}(z)\right| \quad(j=2,3, \ldots)
$$


The zeros $\left\{a_{n}\right\}_{1}^{\infty}$ in Theorem 4 may not increase as rapidly as $\left\{\left|z_{n}\right|\right\}_{1}^{\infty}$ in (7.2) and yet the derivatives of the corresponding canonical product satisfy a sharper inequality (1.7). Note however that the zeros $\left\{a_{n}\right\}_{1}^{\infty}$ are all real and positive.

\section{REFERENCES}

1. R. P. Boas, Entire functions, Academic Press, New York, 1954. MR 16, 914.

2. Fred Gross, Entire functions of bounded index, Proc. Amer. Math. Soc. 18 (1967), 974-980. MR 36 \#1649.

3. B. Lepson, Differential equations of infinite order, hyper-Dirichlet series and entire functions of bounded index, Proc. Sympos. Pure Math., vol. 11, Amer. Math. Soc., Providence, R. I., 1968, pp. 298-307. MR 38 \#6069.

4. W. J. Pugh and S. M. Shah, On the growth of entire functions of bounded index, Pacific J. Math. (to appear).

5. S. M. Shah, Entire functions of bounded index, Proc. Amer. Math. Soc. 19 (1968), 10171022. MR 38 \#6070.

6. - Entire functions satisfying a linear differential equation, J. Math. Mech. 18 (1968/69), 131-136. MR 37 \#2994.

7. E. C. Titchmarsh, Theory of functions, Oxford Univ. Press, Oxford, 1944.

\section{UNIVERSITY OF KENTUCKY,}

LEXINGTON, KENTUCKY 40506 